\title{
Exchange interaction in itinerant-electron metamagnetism
}

\author{
M. A. Grado-Caffaro and M. Grado-Caffaro \\ C/ Julio Palacios 11, 9-B, 28029-Madrid (Spain) \\ E-mail:ma.grado-caffaro@sapienzastudies.com
}

Received: May 2, 2017

\begin{abstract}
The exchange interaction in itinerant-electron metamagnetism is investigated theoretically. In fact, by considering spin-up and spin-down electrons in an itinerant-electron metamagnetic gas in the presence of an external magnetic field, we show that the difference between the Fermi energies of the spin-up and spin-down electrons equals, up to a multiplicative constant, the absolute value of the matrix element of the Hamiltonian operator relative to the interaction in question. Furthermore, the Stoner formula for the electronic energy of the gas is used to study the size of the exchange interaction.
\end{abstract}

Key words: Itinerant-electron metamagnetism, exchange interaction, Fermi energy, Stoner formula.

\section{Introduction}

Itinerant-electron metamagnetic systems are certainly very attractive but, at least from the theoretical standpoint, little work has been done to date, on the above subject. Nevertheless, appreciable experimental work has been published [1-10]. In this respect, consider, for instance, cobalt-based compounds and alloys. But, however, there are a number of open relevant questions which should be elucidated. Among these questions, we can mention key issues related to magnetic susceptibility and exchange interaction energy. In particular, exchange interaction in itinerant-electron metamagnetic systems plays a significant role. Within this context, we want to remark what means metamagnetism so that metamagnetism may be understood as "beyond magnetism". This, say, singular phenomenon presents some very interesting features which must be investigated theoretically by using powerful analytical tools.

We will start from regarding the role of spin-up and spin-down electrons as well as the role played by their respective Fermi levels. In this framework, the Stoner formula for the electronic energy of an itinerant-electron metamagnetic gas, in the presence of an external magnetic field, will be considered in order to evaluate the size of the exchange 
interaction energy between spin-up electrons and spin-down ones. We will show that the absolute value of the matrix element associated with the Hamiltonian operator corresponding to the exchange interaction (of course, repulsive) in question coincides roughly with the difference between the Fermi energy of the spin-up electrons and the Fermi energy of the spin-down electrons. Our approach is certainly unprecedented and opens new ways to tackle fundamental problems which, until now, have been treated with some inaccuracy. We may say that the present approach is very valuable from a strict mathematical-physics standpoint, providing tangible results whose usefulness is evident. On the other hand, approaches similar to our formulation could be, perhaps, suitable to tackle some interesting questions upon the interaction between superconductivity and magnetism $[11,12]$.

\section{Theory}

Let us consider an itinerant-electron metamagnetic gas, at zero absolute temperature, in the presence of an external magnetic field. We regard the above gas as a two-band system so that one of the two bands is formed by spin-up electrons and the other band is formed by spin-down ones. Within this context, the well-known Stoner formula, which gives the electronic energy of the gas, is as follows (see, for instance, [13]):

$$
\epsilon(W)=\int_{-W}^{E_{F 1}} E g(E, W) d E+\int_{-W}^{E_{F 2}} E g(E, W) d E-\frac{\chi B^{2}}{4 \mu^{2}}\left[\chi J(W)+4 \mu_{B}\right]
$$

where $E$ denotes energy, $g$ stands for electronic density of states (DOS), $W$ is half energybandwidth, $E_{F 1}$ and $E_{F 2}$ are the Fermi levels of the spin-up and spin-down bands, respectively, $\chi$ designates dc magnetic susceptibility, $J$ is the exchange interaction energy between spin-up and spin-down electrons, $B$ is the magnitude of the induction (flux density) of the magnetic field, $\mu_{B}$ is the Bohr magneton, and $\mu$ is the dc magnetic permeability. Note that the exchange interaction depends upon $W$. In turn, $W$ depends on volume. In view of this, by virtue of eq. (1), it is clear that the electronic energy of the gas depends also on volume (magneto-volume) [13-15]. In fact, we have [16]:

$$
W(V)=W\left(V_{0}\right) \exp \left[-\alpha\left(V-V_{0}\right) / V_{0}\right]
$$

where $V$ denotes volume and $\alpha$ is a constant such that $1 \leq \alpha \leq 5 / 3$.

We have said that the gas is considered as a system formed of, say, two subsystems, namely, the spin-up electron band and spin-down electron one. We are interested in determining the energy relative to the exchange between spin-up electrons and spin-down ones. On the other hand, one may assume the electrons in each band as non-interacting particles so the one-electron approximation in each band is valid. Therefore, by these considerations, the exchange interaction energy (as a strictly positive quantity) reads [17]:

$$
J(W)=\frac{\gamma(W)\left(E_{F 1}-E_{F 2}\right)^{2}}{E_{1}-E_{2}}
$$

where $\gamma(W)$ is a special (non-negative) $W$-dependent parameter, $E_{1}$ being the energy of a spin-up electron and $E_{2}$ being the energy of a spin-down electron $\left(E_{1}>E_{2}\right)$. On the other hand, we have that $E_{F 1}>E_{F 2}$. 
We can also write [18-20]:

$$
J(W)=\frac{\left|\left\langle\psi_{1}(W)|\hat{H}| \psi_{2}(W)\right\rangle\right|^{2}}{E_{1}-E_{2}}
$$

where $\hat{H}$ is the corresponding interaction Hamiltonian operator.

Equating formulas (3) and (4), it follows that, up to a parameter which only depends on $W, E_{F 1}-E_{F 2}$ equals the absolute value of the matrix element associated with the exchange interaction. The above parameter may be regarded as constant for small $W$ variations. On the other side, replacing (2) and (3) into (1), we can arrive at the expression of the electronic energy in terms of the magneto-volume and Fermi levels of the spin-up and spin-down bands, knowing, of course, the DOS, that is, $g(E, W)$ which was taken as elliptic in [13] but this is not realistic. By examining formula (1), one can see that for high magnetic external fields and relatively large exchange interaction the electronic energy of the metamagnetic gas can be negative. In addition, we may see that this energy can be equal to zero or very small if the magnetic field is relatively weak regardless of the size of the exchange interaction.

If the exchange interaction is small enough, one has that $E_{F 1} \approx E_{F 2}$ (see relationship (3)). If, moreover, these Fermi levels are sufficiently small and $W$ is also sufficiently small, then the difference between a small positive quantity (as $E_{F 1}$ or $E_{F 2}$ ) and a negative quantity (as $-W$, of small absolute value) can be negligible. Consequently, under the above conditions, it is clear that the two integrals in eq. (1) can be negligible so the electronic energy can be negative for $B \neq 0$ since we must take into account the second negative term with absolute value equal to $\chi \mu_{B} B^{2} / \mu^{2}$ in eq. (1). Notice that the first (negative) term can have very small absolute value because a sufficiently small exchange interaction has been assumed.

\section{Conclusion}

We have investigated exchange interaction in a relevant type of magnetism, namely, itinerant-electron metamagnetism in solids. As a matter of fact, we have elaborated a model which, although apparently simple, provides useful information on the exchange interaction in metamagnetism and its implications on certain aspects of the phenomenon in question related to the electronic energy of an itinerant-electron metamagnetic solid (let us regard formula (1)). At this point, it is worth to note that either electronic energy or lattice energy depends on magneto-volume (the total energy of the solid is the sum of the electronic energy and the lattice energy). On the other hand, we wish to remark the importance of evaluating exchange interaction in metamagnetic solids. In this respect, by

eq. (4), note that, if this interaction is weak enough, then the vectors $\left\langle\psi_{1}\right|$ and $\hat{H}\left|\psi_{2}\right\rangle$ are mutually orthogonal.

\section{References}

[1] T. Goto, K. Fukamichi, H. Yamada, Physica B 300, 167-185 (2001).

[2] T. Goto, H. Katori, T. Aruga Sakakibara, H. Mitamura, K. Fukamichi, K. Murata, J. Appl. Phys. 76, 6682-6687 (1994). 
[3] J. Kubler, Theory of itinerant electron metamagnetism (Oxford University Press, Oxford, 2009).

[4] K. Fukamichi, Itinerant-electron metamagnetism, in Handbook of advanced magnetic materials, Y. Liu, D. J. Sellmyer, D. Shindo (Eds.), pp. 683-744 (Springer-Verlag, 2006).

[5] Y. Nishiyama, S. Hirooka, Phys. Rev. B 56, 7793 (1997).

[6] G. R. Stewart, Rev. Mod. Phys. 73, 797-855 (2001).

[7] C. M. Varma, L. Zhu, Phys. Rev. Lett. 96, 036405 (2006).

[8] D. P. Kozlenko, E. Burzo, P. Vlaic, S. E. Kichanov, A. V. Rutkauskas, B. N. Savenko, Sci. Rep. 5, 8620 (2015).

[9] B. S. Shivaram, B. Dorsey, D. G. Hinks, P. Kumar, Phys. Rev. B 89, 161108 (R) (2014).

[10] B. S. Shivaram, D. G. Hinks, M. B. Maple, M. A. de Andrade, P. Kumar, Phys. Rev. B 89, 241107 (R) (2014).

[11] S. P. Kruchinin, Yu. Dzezherya, J. Supercond. Sci. Technol. 19, 381-384 (2006).

[12] S. P. Kruchinin, H. Nagao, S. Aono, Modern aspects of superconductivity-theory of superconductivity, pp.166-181 (World Scientific Pub., 2011).

[13] N. H. Duc, D. Givord, C. Lacroix, C. Pinettes, Europhys. Lett. 20, 47-52 (1992).

[14] J. Inoue, M. Shimizu, J. Phys. F: Metal Phys. 12, 1811 (1982).

[15] E. Gratz, A.S. Markosyan, J. Phys.: Condens. Matter 13, R385 (2001).

[16] J. C. Slater, G. Koster, Phys. Rev. 94, 1498 (1954).

[17] M. A. Grado-Caffaro, M. Grado-Caffaro, Zeitschrift für Naturforschung A 72(5), 463467 (2017).

[18] T. J. Han, In. M. Kim, S. H. Choh, Can. J. Phys. 64, 252-255 (1986).

[19] M. A. Grado-Caffaro, M. Grado-Caffaro, Acta Phys. Pol. A 128, 394-398 (2015).

[20] M. A. Grado-Caffaro, M. Grado-Caffaro, Z. Naturforsch. A (Phys. Sci.) 71, 311-314 (2016). 